GEOMETRY AND ANALYSIS ON LIE GROUPS

BANACH CENTER PUBLICATIONS, VOLUME 55

INSTITUTE OF MATHEMATICS

POLISH ACADEMY OF SCIENCES

WARSZAWA 2002

\title{
THE WEYL ALGEBRA, SPHERICAL HARMONICS, AND HAHN POLYNOMIALS
}

\author{
EWA GNATOWSKA \\ Department of Mathematical Methods of Physics, Faculty of Physics, University of Warsaw \\ Hoża 74, 00-682 Warszawa, Poland \\ E-mail: gnatowsk@fuw.edu.pl \\ ALEKSANDER STRASBURGER \\ Institute of Mathematics, University of Biatystok, Akademicka 2, 15-267 Biatystok, Poland \\ E-mail: alekstra@math.uwb.edu.pl \\ and \\ Department of Mathematical Methods of Physics, Faculty of Physics, University of Warsaw \\ Hoża 74, 00-682 Warszawa, Poland \\ E-mail: alekstra@fuw.edu.pl
}

\begin{abstract}
In this article we apply the duality technique of R. Howe to study the structure of the Weyl algebra. We introduce a one-parameter family of "ordering maps", where by an ordering map we understand a vector space isomorphism of the polynomial algebra on $\mathbb{R}^{2 d}$ with the Weyl algebra generated by creation and annihilation operators $a_{1}, \ldots, a_{d}, a_{1}^{+}, \ldots, a_{d}^{+}$. Corresponding to these orderings, we construct a one-parameter family of $\mathfrak{s l}_{2}$ actions on the Weyl algebra, which enables us to define and study certain subspaces of the Weyl algebra the space of Weyl spherical harmonics and the space of "radial polynomials". For the latter we generalize results of Louck and Biedenharn, Bender et al., and Koornwinder describing the radial elements in terms of continuous Hahn polynomials of the number operator.
\end{abstract}

1. Introduction. This article reports on an investigation (still in progress) of the Weyl algebra with the aid of R. Howe's duality technique. More specifically, we attempt to transfer the information obtained by considering the action on the (commutative) polynomial algebra on $\mathbb{R}^{2 d}$ of a triple of classical operators (consisting of the Laplacian, Euler operator and multiplication by the square of the radius and giving rise to an $\mathfrak{s l}_{2}$ action on this algebra) to the case of the (noncommutative) Weyl algebra with $2 d$

2000 Mathematics Subject Classification: Primary 81R10, 33C55; Secondary 16W25, 33C45, $33 \mathrm{C} 80$.

Received 22 August 2001; revised 28 September 2001.

The paper is in final form and no version of it will be published elsewhere. 
generators - see the beginning of Section 3 for the definition of the Weyl algebra. On this route one is inevitably confronted with the renowned "ordering problem of quantum mechanics", cf. e.g. [17, 2, 3]. We deal with this problem in a wider context by introducing a one-parameter family of "orderings", by which we understand a family of vector space isomorphisms of the polynomial algebra with the Weyl algebra. Corresponding to these orderings, we construct a one-parameter family of $\mathfrak{s l}_{2}$ representations acting on the Weyl algebra, whose action we employ to decompose the algebra.

Two subspaces of the Weyl algebra stand out in our analysis - the subspace of "Weyl spherical harmonics" and the (in a certain sense complementary) subspace of "radial" polynomials, which are polynomials of a single element, the so-called number operator. In this paper we concentrate on the latter, emphasizing the connection with the problems of the special function theory. This is the subject of the second part of the paper, where we describe properties of these radial polynomials in various orderings. In particular we identify the elements of a natural basis of the space with Hahn polynomials of the shifted number operator. This is a generalization of results obtained earlier by Lohe, Biedenharn and Louck [11], Bender, Mead, and Pinsky [2], and Koornwinder [10], among others.

2. Preliminaries on the polynomial algebra in even dimension. Consider the real cartesian space of even dimension $\mathbb{R}^{2 d}, d \geq 1$, with coordinates denoted by $x_{1}, \ldots$, $x_{d}, \xi_{1}, \ldots, \xi_{d}$. Let $P=P_{\mathbb{C}}\left(\mathbb{R}^{2 d}\right)$ denote the polynomial algebra with complex coefficients over $\mathbb{R}^{2 d}$ and $P^{k}=P_{\mathbb{C}}^{k}\left(\mathbb{R}^{2 d}\right)$ its subspace consisting of homogeneous polynomials of degree $k$. Setting $z_{j}=x_{j}+i \xi_{j}$ and $\bar{z}_{j}=x_{j}-i \xi_{j}$ for $j=1, \ldots, d$, where here and everywhere in the sequel $i=\sqrt{-1}$, we can regard $P$ as the polynomial algebra with respect to the coordinate functions $z_{1}, \ldots, z_{d}$ and their conjugates $\bar{z}_{1}, \ldots, \bar{z}_{d}$. Thus, using the usual multi-index notation we shall write its elements in the form

$$
p(z, \bar{z})=\sum_{\alpha, \beta} p_{\alpha, \beta} z^{\alpha} \bar{z}^{\beta},
$$

where $z=\left(z_{1}, \ldots, z_{d}\right), \bar{z}=\left(\bar{z}_{1}, \ldots, \bar{z}_{d}\right) \in \mathbb{C}^{d}$ and $p_{\alpha, \beta} \in \mathbb{C}$. In particular,

$$
r^{2}=\sum_{j=1}^{d} x_{j}^{2}+\sum_{j=1}^{d} \xi_{j}^{2}=\sum_{j=1}^{d} z_{j} \bar{z}_{j}
$$

is the square-of-the-radius function (euclidean length squared) on $\mathbb{R}^{2 d}$.

Similarly, setting as usual

$$
\frac{\partial}{\partial z_{j}}=\frac{1}{2}\left(\frac{\partial}{\partial x_{j}}-i \frac{\partial}{\partial \xi_{j}}\right), \quad \frac{\partial}{\partial \bar{z}_{j}}=\frac{1}{2}\left(\frac{\partial}{\partial x_{j}}+i \frac{\partial}{\partial \xi_{j}}\right),
$$

one can express in the complex form the euclidean Laplace operator (Laplacian)

$$
\Delta=\sum_{j=1}^{d} \frac{\partial^{2}}{\partial x_{j}^{2}}+\sum_{j=1}^{d} \frac{\partial^{2}}{\partial \xi_{j}^{2}}=4 \sum_{j=1}^{d} \frac{\partial^{2}}{\partial z_{j} \partial \bar{z}_{j}}
$$

and the Euler operator

$$
\widetilde{E}=\sum_{j=1}^{d} x_{j} \frac{\partial}{\partial x_{j}}+\sum_{j=1}^{d} \xi_{j} \frac{\partial}{\partial \xi_{j}}=\sum_{j=1}^{d} z_{j} \frac{\partial}{\partial z_{j}}+\sum_{j=1}^{d} \overline{z_{j}} \frac{\partial}{\partial \bar{z}_{j}} .
$$


Along with the usual action of the orthogonal group $\mathbf{O}(2 d)$ on $P$, we shall be concerned with the action of the unitary group $\mathbf{U}(d)$ on $P$ which is obtained by extending the action on generators given by

$$
z \mapsto g z, \quad \bar{z} \mapsto \bar{g} \bar{z}, \quad g \in \mathbf{U}(d),
$$

where $z=\left(z_{1}, \ldots, z_{d}\right)$ and $\bar{z}=\left(\bar{z}_{1}, \ldots, \bar{z}_{d}\right)$ are regarded as column vectors. Recall that the contragredient matrix $\left(g^{t}\right)^{-1}$ of any $g \in \mathbf{U}(d)$ satisfies $\left(g^{t}\right)^{-1}=\bar{g}$, the bar denoting complex conjugation (taken entry-wise). These two actions are related by the natural embedding of $\mathbf{U}(d)$ into $\mathbf{O}(2 d)$.

Modifying slightly the above differential operators and supplementing them by the multiplication by the square of the radius one obtains a triple of endomorphisms inducing an action of the Lie algebra $\mathfrak{s l}_{2}$ on $P$. In fact, it can be easily checked that by setting

$$
R p=r^{2} p, \quad L p=\frac{1}{4} \Delta p, \quad E p=(\widetilde{E}+d) p, \quad \text { for } p \in P,
$$

(here $\widetilde{E}+d$ is the symmetrized Euler operator) we obtain commutation rules for the standard generators of the Lie algebra $\mathfrak{s l}_{2}$, i.e.

$$
[R, L]=-E, \quad[E, R]=2 R, \quad[E, L]=-2 L .
$$

It can be verified that this latter action commutes with the action of $\mathbf{O}(2 d)$. A remarkable although elementary application of Howe's duality principle based on using the above actions is the analysis of the structure of the algebra $P$ (cf. [8], p. 118), which gives a large part of the classical theory of spherical harmonics. In particular one arrives this way at the decomposition $P=J \otimes H$, where $J \subset P$ is the subalgebra of $\mathbf{O}(2 d)$ invariant polynomials, known to be the subalgebra generated by $r^{2}$, and $H=H\left(\mathbb{R}^{2 d}\right)=\{p \in P \mid \Delta p=0\}$ is the subspace of harmonic polynomials in $P$. The isomorphism is obtained via multiplication. Consequently each homogeneous polynomial $p \in P$ can be decomposed into a sum of products of homogeneous harmonic polynomials with powers of the radius-square;

$$
p(z, \bar{z})=\sum_{j=0}^{\left[\frac{1}{2} \operatorname{deg} p\right]} r^{2 j} h_{j}(z, \bar{z}),
$$

where $h_{j}$ are homogeneous harmonic polynomials of degree $\operatorname{deg} p-2 j$. (See e.g. [5] for a derivation of this decomposition along classical lines).

Furthermore, the following facts can be obtained within this approach.

Let $H^{k} \subset H$ denote the subspace of homogeneous harmonic polynomials of degree $k$.

a) For each $k \in \mathbb{N}$ the subspace $H^{k}$ is irreducible under the natural action of $\mathbf{O}(2 d)$ and for any $j \neq k$ the actions on $H^{j}$ and $H^{k}$ are not equivalent. Therefore the direct sum decomposition $H=\oplus_{j=0}^{\infty} H^{j}$ is multiplicity free.

b) For each $m \in \mathbb{N}$ the space $P^{m}$ decomposes into a direct sum of $\mathbf{O}(2 d)$ irreducible subspaces as

$$
P^{m}=\bigoplus_{j=0}^{\left[\frac{1}{2} m\right]} r^{2 j} H^{m-2 j} .
$$

c) Let $P\left(\mathbb{R}^{2 d} ; H^{k}\right)$ be the sum of all $\mathbf{O}(2 d)$ irreducible subspaces of $P$ equivalent to $H^{k}$; it is called the isotypic subspace of type $H^{k}$. Then the multiplication 


$$
J \otimes H^{k} \rightarrow P\left(\mathbb{R}^{2 d} ; H^{k}\right) ; \quad p\left(r^{2}\right) \otimes h(z, \bar{z}) \mapsto p\left(r^{2}\right) \cdot h(z, \bar{z})
$$

induces an equivariant isomorphism of $P\left(\mathbb{R}^{2 d} ; H^{k}\right)$ with $J \otimes H^{k}$.

As the actions of $\mathfrak{s l}_{2}$ and $\mathbf{O}(2 d)$ commute with each other, it makes sense to consider their joint action - perhaps more properly one should speak of the action of the direct product of the Lie algebras $\mathfrak{s l}_{2}$ and $\mathfrak{o}(2 d)$ on $P$. It can be shown that the space $P\left(\mathbb{R}^{2 d} ; H^{k}\right)$ is irreducible under this joint action. By transfering the action of $\mathfrak{s l}_{2}$ to the algebra of invariants $J=P\left[r^{2}\right]$ by means of the multiplication map (2.5) one obtains the action which is (algebraically) equivalent to that of one of the lowest weight representations of $\mathfrak{s l}_{2}$ - the lowest weight (with respect to $E$ ) being equal to $(k+d)$. As shown in [13], the weight vectors for this action can be identified with certain Laguerre polynomials.

Lastly, it is known that under restriction to $\mathbf{U}(d) \subset \mathbf{O}(2 d)$ the irreducible $\mathbf{O}(2 d)$-space $H^{k}$ splits into the direct sum of $\mathbf{U}(d)$-irreducible subspaces,

$$
H^{k}=\bigoplus_{m+n=k} H^{(n, m)}
$$

where $H^{(n, m)}$ denotes the space of homogeneous polynomials of bi-degree $(n, m)$. We recall that $p(z, \bar{z}) \in H^{(n, m)} \Leftrightarrow p(\lambda z, \bar{\lambda} \bar{z})=\lambda^{n} \bar{\lambda}^{m} p(z, \bar{z})$. Consequently, the isotypic subspaces of type $H^{(n, m)}$ in $P$ are of the form $J \cdot H^{(n, m)}$, and

$$
P\left(\mathbb{R}^{2 d} ; H^{k}\right)=\bigoplus_{m+n=k} J \cdot H^{(n, m)} .
$$

For the explicit procedure of obtaining the decomposition (2.6), in particular for the formulae expressing projections onto subspaces of the given bi-degree, we refer to [9].

3. Construction of an $\mathfrak{s l}_{2}$ action on the Weyl algebra. In this section we shall describe a method of transferring the results concerning the polynomial algebra described at the end of the preceding section to the case of the Weyl algebra.

Usually, by the Weyl algebra with $2 d$ generators $\mathcal{W}=\mathcal{W}_{2 d}$ one means the associative algebra with unit $I$ generated by $2 d$ elements $a_{1}, \ldots, a_{d}, a_{1}^{+}, \ldots, a_{d}^{+}$subject to the relations (called Canonical Commutation Relations - abbreviated CCR in the sequel)

$$
a_{j} a_{k}^{+}-a_{k}^{+} a_{j}=\delta_{k j} I, \quad a_{j} a_{k}-a_{k} a_{j}=0, \quad a_{k}^{+} a_{j}^{+}-a_{k}^{+} a_{j}^{+}=0, \quad 1 \leq j, k \leq d .
$$

We shall employ the following standard notation from the theory of associative algebras: for arbitrary $x, y \in \mathcal{W}$ we shall denote by $[x, y]=x y-y x$ the commutator of $x$ and $y$ and by $\{x, y\}=x y+y x$ their anticommutator. For $x \in \mathcal{W}$ the adjoint map $\operatorname{ad}(x): \mathcal{W} \rightarrow \mathcal{W}$ is defined by setting $\operatorname{ad}(x) w=[x, w]$ for $w \in \mathcal{W}$ - as is well-known, $\operatorname{ad}(x)$ is a derivation of $\mathcal{W}$.

Let us recall that for many questions it is more convenient to realize the abstractly defined Weyl algebra as the algebra of partial differential operators with polynomial coefficients, considered initially as operators acting as on the Schwartz space $\mathcal{S}\left(\mathbb{R}^{d}\right)$, and regarded, when need arises, as densely defined operators in $L^{2}\left(\mathbb{R}^{d}, d \lambda(x)\right)$ - the space of square integrable functions on $\mathbb{R}^{d}$ with respect to the Lebesgue measure. This identification is obtained by the standard assignment

$$
a_{j}=\frac{1}{\sqrt{2}}\left(x_{j}+\frac{\partial}{\partial x_{j}}\right), \quad a_{j}^{+}=\frac{1}{\sqrt{2}}\left(x_{j}-\frac{\partial}{\partial x_{j}}\right) .
$$


Note that $a_{j}^{*}=a_{j}^{+}$, where the adjoint * operation is understood in the sense of the formal adjoint of a differential operator. In quantum physics the operators $a_{j}, a_{j}^{+}$are known as annihilation and creation operators, respectively. For the present work however, it is the algebraic structure of the Weyl algebra $\mathcal{W}$ that matters.

Given $a \in \mathcal{W}$ we denote by $L_{a}$ the map $\mathcal{W} \rightarrow \mathcal{W}$ obtained by the left multiplication with $a$ and by $L_{a}^{+}$the map corresponding to the left multiplication by $a^{+}$. The symbols $R_{a}, R_{a}^{+}$will have analogous meanings with respect to the right multiplication. By taking convex combinations of left and right multiplication operators we shall be able to construct a family of endomorphisms of $\mathcal{W}$ which will serve as a replacement for the operators of multiplication by coordinates and their complex conjugates in the polynomial algebra.

Definition 1. For a given $a \in \mathcal{W}$ and $q \in[0,1]$ we define $\left({ }^{1}\right)$ endomorphisms $M_{a}$ and $M_{a}^{+}$of $\mathcal{W}$ by the formulae

$$
\begin{aligned}
M_{a} & =(1-q) L_{a}+q R_{a}=L_{a}-q \operatorname{ad}(a), \\
M_{a}^{+} & =q L_{a}^{+}+(1-q) R_{a}^{+}=L_{a}^{+}-(1-q) \operatorname{ad}\left(a^{+}\right) .
\end{aligned}
$$

Let us explicitly point out to the meaning of this definition for the special values $q=0, \frac{1}{2}, 1$ of the parameter. For $q=0, M_{a}$ is the left multiplication by $a$, while $M_{a}^{+}$is the right multiplication by $a^{+}$. For $q=1$ these roles are reversed, while for $q=\frac{1}{2}$ each is equal to $1 / 2$ of the respective anticommutator.

LEMMA 1. Let $\left\{a_{1}, \ldots, a_{d}, a_{1}^{+}, \ldots, a_{d}^{+}\right\}$be a set of generators of the Weyl algebra and put $M_{j}=M_{a_{j}}, M_{j}^{+}=M_{a_{j}}^{+}, j=1, \ldots, d$. Then $\left\{M_{1}, \ldots, M_{d}, M_{1}^{+}, \ldots, M_{d}^{+}\right\}$is a commutative family of endomorphisms of $\mathcal{W}$.

Proof. In view of CCR it is clear that the $M_{j}$ 's $\left(M_{j}^{+}\right.$'s respectively) commute, and that $\left[M_{j}, M_{k}^{+}\right]=0$ for $j \neq k$. This last relation is also true for $j=k$. In fact

$$
\left[M_{j}, M_{j}^{+}\right]=(1-q) q\left(\left[L_{a_{j}}, L_{a_{j}}^{+}\right]+\left[R_{a_{j}}, R_{a_{j}}^{+}\right]\right)=(1-q) q(I-I)=0 .
$$

Thus employing the multi-index notation for the family $\left\{M_{1}, \ldots, M_{d}, M_{1}^{+}, \ldots, M_{d}^{+}\right\}$, i.e. writing $M^{\alpha} M^{+\beta}=M_{1}^{\alpha_{1}} \ldots M_{d}^{\alpha_{d}} M_{1}^{+\beta_{1}} \ldots M_{d}^{+\beta_{d}}$ for $\alpha, \beta \in \mathbb{Z}_{+}^{d}$, we have by virtue of the lemma

$$
M^{\alpha} M^{+\beta} \cdot M^{\alpha^{\prime}} M^{+\beta^{\prime}}=M^{\left(\alpha+\alpha^{\prime}\right)} M^{+\left(\beta+\beta^{\prime}\right)} .
$$

Lemma 2. The $\operatorname{map} \mathcal{O}_{q}: P\left(\mathbb{R}^{2 d}\right) \rightarrow \mathcal{W}_{2 d}$ defined by

$$
\mathcal{O}_{q}\left(z^{\alpha} \bar{z}^{\beta}\right)=M^{\alpha} M^{+\beta} I
$$

is a vector space isomorphism such that

$$
\begin{array}{cc}
\mathcal{O}_{q}\left(z_{j} p\right)=M_{j} \mathcal{O}_{q}(p), & \mathcal{O}_{q}\left(\overline{z_{j}} p\right)=M_{j}^{+} \mathcal{O}_{q}(p), \\
\mathcal{O}_{q}\left(\frac{\partial p}{\partial z_{j}}\right)=-\operatorname{ad}\left(a_{j}^{+}\right) \mathcal{O}_{q}(p), & \mathcal{O}_{q}\left(\frac{\partial p}{\partial \overline{z_{j}}}\right)=\operatorname{ad}\left(a_{j}\right) \mathcal{O}_{q}(p) .
\end{array}
$$

Proof. The first pair of relations is obvious by the definition of $\mathcal{O}_{q}$ and Lemma 1. The second pair, concerning derivations, follows by routine calculation.

$\left({ }^{1}\right)$ The condition $q \in[0,1]$ turns out to be inessential and will be removed in further publications. 
Definition 2. The map $\mathcal{O}_{q}: P\left(\mathbb{R}^{2 d}\right) \rightarrow \mathcal{W}_{2 d}$ defined for $q \in[0,1]$ by the equation (3.5) will be called the $q$-ordering map.

Since the subalgebras of $\mathcal{W}$ generated by pairs $\left\{a_{l}, a_{l}^{+}\right\},\left\{a_{k}, a_{k}^{+}\right\}$with $k \neq l$, commute with each other, the elements (3.5) can be written in the factorized form,

$$
\mathcal{O}_{q}\left(z^{\alpha} \bar{z}^{\beta}\right)=\prod_{l=1}^{d} B_{\beta_{l}}^{\alpha_{l}}\left(a_{l}, a_{l}^{+}\right)
$$

where for nonnegative integers $k, j$ we have set

$$
B_{j}^{k}\left(a_{l}, a_{l}^{+}\right)=\sum_{s=0}^{k}\left(\begin{array}{l}
k \\
s
\end{array}\right) q^{k-s}(1-q)^{s} a_{l}^{s}\left(a_{l}^{+}\right)^{j} a_{l}^{k-s} .
$$

In the special cases mentioned above the formula (3.8) specializes to the following well-known constructions.

a) For $q=0$ it gives the so-called normal (Wick) ordering

$$
\mathcal{O}_{0}\left(z^{\alpha} \bar{z}^{\beta}\right)=a^{\alpha}\left(a^{+}\right)^{\beta}
$$

with all $a_{j}$ (annihilation operators) on the left and all $a_{j}^{+}$(creation operators) on the right.

b) For $q=1$ the roles of $a_{j}$ and $a_{j}^{+}$are reversed and $\mathcal{O}_{1}$ gives the anti-normal (anti-Wick) ordering

$$
\mathcal{O}_{1}\left(z^{\alpha} \bar{z}^{\beta}\right)=a^{+\beta} a^{\alpha} .
$$

c) The case $q=\frac{1}{2}$ corresponds to the so-called Weyl, or symmetric, ordering.

All these assertions are evident on the basis of (3.2). Further, let us observe that our elements $B_{j}^{k}$ are related to the basis elements $P_{m}^{j}$ introduced for the case of one degree of freedom $(d=1)$ by Louck and Biedenharn in [4]. In fact, $B_{j}^{k}$ is proportional to

$$
P_{\frac{j+k}{2}}^{\frac{j-k}{2}}\left(a, a^{+}\right)=2^{\frac{j-k}{2}} \sqrt{\frac{k !}{j !}} \sum_{l=0}^{k} \frac{a^{l}\left(a^{+}\right)^{j} a^{k-l}}{l !(j-l) !} .
$$

Since the $q$-ordering map $\mathcal{O}_{q}$ is for each value of $q$ an isomorphism of vector spaces, there exist endomorphisms of the Weyl algebra, which we shall denote $\mathcal{R}_{q}, \mathcal{L}_{q}$, and $\mathcal{E}_{q}$ (for simplicity we suppress the index $q$ writing $\mathcal{R}=\mathcal{R}_{q}$ etc., when no confusion can arise), such that the following equalities hold:

$$
\mathcal{O}_{q} R=\mathcal{R}_{q} \mathcal{O}_{q}, \quad \mathcal{O}_{q} L=\mathcal{L}_{q} \mathcal{O}_{q}, \quad \mathcal{O}_{q} E=\mathcal{E}_{q} \mathcal{O}_{q}
$$

This can be conveniently summarised in the form of commutative diagrams

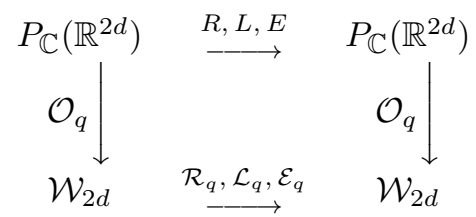

From (2.2) and (3.6-3.7) we can deduce explicit expressions for $\mathcal{R}_{q}, \mathcal{L}_{q}$ and $\mathcal{E}_{q}$. 
Lemma 3. The endomorphisms $\mathcal{R}_{q}, \mathcal{L}_{q}, \mathcal{E}_{q}$ of the Weyl algebra $\mathcal{W}_{2 d}$ are given by the following formulae:

$$
\begin{aligned}
& \mathcal{R}_{q} w=(1-q)^{2} \sum_{j=1}^{d} a_{j} w a_{j}^{+}+q(1-q) \sum_{j=1}^{d}\left(w a_{j}^{+} a_{j}+a_{j} a_{j}^{+} w\right)+q^{2} \sum_{j=1}^{d} a_{j}^{+} w a_{j}, \\
& \mathcal{L}_{q} w=-\sum_{j=1}^{d} \operatorname{ad}\left(a_{j}\right) \operatorname{ad}\left(a_{j}^{+}\right) w \\
& \mathcal{E}_{q} w=-\sum_{j=1}^{d}\left\{(1-q)\left(a_{j}\left[a_{j}^{+}, w\right]-\left[a_{j}, w\right] a_{j}^{+}\right)+q\left(\left[a_{j}^{+}, w\right] a_{j}-a_{j}^{+}\left[a_{j}, w\right]\right)\right\}+d w .
\end{aligned}
$$

Note that $\mathcal{L}_{q}$ does not depend on $q$ and moreover, $\operatorname{since} \operatorname{ad}\left(a_{j}\right) \operatorname{ad}\left(a_{j}^{+}\right)=\operatorname{ad}\left(a_{j}^{+}\right) \operatorname{ad}\left(a_{j}\right)$, we also have $\mathcal{L}_{q}=-\sum_{j=1}^{d} \operatorname{ad}\left(a_{j}^{+}\right) \operatorname{ad}\left(a_{j}\right)$.

For the case of the Weyl (symmetric) ordering, i.e. for $q=\frac{1}{2}$, we shall employ the notation $\mathcal{R}_{s}, \mathcal{L}_{s}, \mathcal{E}_{s}$ for these operators. Note that they are given by fairly simple expressions (recall $\{a, b\}$ stands for the anticommutator of $a, b \in \mathcal{W}$ ):

$$
\begin{aligned}
& \mathcal{R}_{s} w=\frac{1}{4} \sum_{j=1}^{d}\left\{a_{j},\left\{a_{j}^{+}, w\right\}\right\}, \\
& \mathcal{L}_{s} w=-\sum_{j=1}^{d}\left[a_{j},\left[a_{j}^{+}, w\right]\right], \\
& \mathcal{E}_{s} w=\sum_{j=1}^{d}\left(a_{j} w a_{j}^{+}-a_{j}^{+} w a_{j}\right) .
\end{aligned}
$$

Applying successively the defining relation (3.9) to the commutation relations (2.3), we see that the endomorphisms $\mathcal{R}_{q}, \mathcal{L}_{q}$ and $\mathcal{E}_{q}$ of the Weyl algebra also satisfy the commutation relations of the $\mathfrak{s l}_{2}$ Lie algebra, namely

$$
\left[\mathcal{R}_{q}, \mathcal{L}_{q}\right]=-\mathcal{E}_{q}, \quad\left[\mathcal{E}_{q}, \mathcal{R}_{q}\right]=2 \mathcal{R}_{q}, \quad\left[\mathcal{E}_{q}, \mathcal{L}_{q}\right]=-2 \mathcal{L}_{q}
$$

(which can also be checked by brute force calculation). This leads us to the following important result.

Corollary 1. The q-ordering map $\mathcal{O}_{q}: P\left(\mathbb{R}^{2 d}\right) \rightarrow \mathcal{W}_{2 d}$ is an intertwining map for actions of the Lie algebra $\mathfrak{s l}_{2}$ realized by the triples $\{R, L, E\}$ and $\left\{\mathcal{R}_{q}, \mathcal{L}_{q}, \mathcal{E}_{q}\right\}$.

4. The analysis of radial Weyl polynomials. We now apply the idea of $\mathrm{R}$. Howe to use the action of $\mathfrak{s l}_{2}$ determined by $\left\{\mathcal{R}_{q}, \mathcal{L}_{q}, \mathcal{E}_{q}\right\}$ to decompose the Weyl algebra $\mathcal{W}$. Given any homogeneous polynomial $p \in P^{l}$ we can decompose it according to (2.4), so that $p=\sum_{k=0}^{[l / 2]} r^{2 k} h_{k}, h_{k} \in H^{l-2 k}$. It follows that

$$
\mathcal{O}_{q}(p)=\sum_{k=0}^{[l / 2]} \mathcal{R}_{q}^{k} \mathcal{O}_{q}\left(h_{k}\right) .
$$

By virtue of the intertwining property (3.10) and (3.12) we obtain: 
Proposition 1. The image of the space $H$ of harmonic polynomials in $P$ under the $q$-ordering $\operatorname{map} \mathcal{O}_{q}$ is independent of $q$. For $w \in \mathcal{W}$ we have

$$
w \in \mathcal{O}_{q}(H) \Leftrightarrow \sum_{j=1}^{d}\left[a_{j},\left[a_{j}^{+}, w\right]\right]=0 .
$$

We shall denote this space by $\mathcal{H}=\mathcal{O}_{q}(H)$ and shall refer to it as the space of Weyl algebra harmonics. We note that Geller in [7] observed, with a very different method and for the particular cases $q=0, \frac{1}{2}, 1$ only, this somewhat surprising fact that the space of Weyl algebra harmonics does not depend on the ordering.

Getting back to (4.1), we see that two factors are essential for understanding the structure of the Weyl algebra: the space $\mathcal{H} \subset \mathcal{W}$ of Weyl harmonics and the action of operators $\mathcal{R}_{q}$ on $\mathcal{W}$. In fact, the equality (4.1) leads to the following result.

Proposition 2. Recall the space of radial polynomials in $P, J=\mathbb{C}\left[r^{2}\right]$, and for $p\left(r^{2}\right)=\sum_{k=0}^{l} c_{k} r^{2 k} \in J$ set $p\left(\mathcal{R}_{q}\right)=\sum_{k=0}^{l} c_{k} \mathcal{R}_{q}^{k}$. Then the map

$$
J \times H \ni(p, h) \rightarrow p\left(\mathcal{R}_{q}\right) \mathcal{O}_{q}(h) \in \mathcal{W}
$$

gives rise to an isomorphism of $J \otimes H$ with $\mathcal{W}$.

In the sequel we shall investigate in detail the image $\mathcal{J}_{q}=\mathcal{O}_{q}(J)=\left\{p\left(\mathcal{R}_{q}\right) I \mid p \in J\right\}$ of the space $J$ of invariant polynomials under the ordering map. Although apparently depending on $q$ through its construction, it will turn out to be the same for all values of $q$ and consist of polynomials of a single element (the number operator given by (4.3) below) of $\mathcal{W}$ - cf. Proposition 3 below.

For each nonnegative integer $k$ we set

$$
\eta_{k}=\eta_{k}[q, d]=\mathcal{R}_{q}^{k} I
$$

so that $\left\{\eta_{k}\right\}$ is a natural basis of $\mathcal{J}_{q}$. Let us write down the explicit form of a few first elements of this sequence. Clearly $\eta_{0}=I$, and after collecting terms in (3.11) and making use of the CCR (3.1) we arrive at

$$
\eta_{1}=\sum_{j=1}^{d} a_{j}^{+} a_{j}+d(1-q) I=N+d(1-q) I .
$$

Here we have denoted by

$$
N=\sum_{j=1}^{d} a_{j}^{+} a_{j}
$$

an element of the Weyl algebra, in physics called the number operator, which plays a rather special role in questions related to the structure of the Weyl algebra, see e.g. $[8,14,15]$. Its relevance to the present situation stems from the fact that the space $\mathcal{J}_{q}$ consists of polynomials of $N$. Before proving this fact, we bring in for illustration the expression of $\eta_{2}$ in terms of $N$. Setting for simplicity $t_{0}=d(1-q)$, it reads as follows:

$$
\eta_{2}(N)=\left(N+t_{0} I\right)^{2}+(1-2 q)\left(N+t_{0} I\right)+q t_{0} I .
$$


Lemma 4. For each $k \in \mathbb{Z}_{+}$there exists a unique polynomial $\omega_{k}(t)=\omega_{k}(q, d ; t)$ of exact degree $k, \omega_{k} \in \mathbb{C}[t]$, such that

$$
\eta_{k}[q, d]=\omega_{k}(N) .
$$

Proof. Clearly $\omega_{0}(t) \equiv 1$ and the form of $\omega_{1}(t)$ and $\omega_{2}(t)$ can be read off from the above formulae. To prove the result in general we proceed by induction with respect to $k$. First note the general relations (the so called pull-through relations)

$$
a_{i} p(N)=p(N+1) a_{i}, \quad a_{i}^{+} p(N)=p(N-1) a_{i}^{+}
$$

valid for any polynomial $p(N)$ of the number operator. It follows that

$$
\mathcal{R}_{q} p(N)=q(1-q)(2 N+d) p(N)+(1-q)^{2}(N+d) p(N+1)+q^{2} N p(N-1) .
$$

A comparison of highest order coefficients in the terms on the right hand side shows that applying $\mathcal{R}_{q}$ to a polynomial in $N$ increases its degree by one.

For completeness we explicitly state the following

Proposition 3. The space $\mathcal{J}_{q}$ is independent of $q$ and consists of polynomials in terms of the number operator $N$. Thus we set

$$
\mathcal{J}=\mathcal{J}_{q}=\mathbb{C}[N]
$$

To determine the form of polynomials $\omega_{k}$ we shall investigate the difference and recurrence equations satisfied by them. We begin with the following observation.

Lemma 5. The space $\mathcal{J}$ is invariant under the action of the triple $\left(\mathcal{R}_{q}, \mathcal{L}_{q}, \mathcal{E}_{q}\right)$ and in fact $\eta_{k}$ given by (4.2), with $k$ ranging over all nonnegative integers, form a weight basis for the $\mathfrak{s l}_{2}$ action on $\mathcal{J}$ so defined. Setting for convenience $\eta_{-1}=0$ this action is expressed by the well-known formulae

$$
\begin{aligned}
\mathcal{R}_{q} \eta_{k} & =\eta_{k+1}, \\
\mathcal{L}_{q} \eta_{k} & =k(k+d-1) \eta_{k-1}, \\
\mathcal{E}_{q} \eta_{k} & =(2 k+d) \eta_{k} .
\end{aligned}
$$

This immediately follows by comparing the definition (4.2) and the corresponding action of $\{R, L, E\}$ on the space of radial polynomials.

By virtue of Lemma 4 we can transfer the action of $\left\{\mathcal{R}_{q}, \mathcal{L}_{q}, \mathcal{E}_{q}\right\}$ to the space of polynomials $\mathbb{C}[t]$. If $\mathcal{X}$ is any of $\mathcal{R}_{q}, \mathcal{L}_{q}, \mathcal{E}_{q}$ we define the map $\widetilde{X}: \mathbb{C}[t] \rightarrow \mathbb{C}[t]$ by setting

$$
(\tilde{X} p)(N)=\mathcal{X} p(N) \text {. }
$$

By the proof of Lemma 4, the right hand side is a uniquely determined polynomial of the number operator $N$.

Employing the classical notation for the forward and backward difference operators, i.e. setting

$$
\Delta \omega(t)=\omega(t+1)-\omega(t), \quad \nabla \omega(t)=\omega(t)-\omega(t-1),
$$

we can state the following. 
LEMmA 6. The action of the triple $\left\{\mathcal{R}_{q}, \mathcal{L}_{q}, \mathcal{E}_{q}\right\}$ on $\mathcal{J}$ induces the following action on $\mathbb{C}[t]$ :

$$
\begin{aligned}
& \widetilde{R_{q}} \omega(t)=(1-q)^{2}(t+d) \Delta \omega(t)-q^{2} t \nabla \omega(t)+(t+d(1-q)) \omega(t), \\
& \widetilde{L_{q}} \omega(t)=(t+d) \Delta \omega(t)-t \nabla \omega(t), \\
& \widetilde{E_{q}} \omega(t)=2(1-q)(t+d) \Delta \omega(t)+2 q t \nabla \omega(t)+d \omega(t) .
\end{aligned}
$$

The proof is a routine calculation based on Lemma 3 and (4.4).

A consequence of the relation

$$
\mathcal{E}_{q} \eta_{k}=\widetilde{E_{q}} \omega_{k}(N)=k \omega_{k}(N)
$$

is a difference equation satisfied by $\omega_{k}$ which reads

$$
q t \nabla \omega_{k}(t)+(1-q)(t+d) \Delta \omega_{k}(t)=k \omega_{k}(t) .
$$

In order to fix a multiplicative constant for $\omega_{k}$ which is not determined by the equation (4.8), we look for an additional relation. This is supplied by taking a suitable combination of operators appearing in Lemma 6 . One notes that the operator $\widetilde{R_{q}}-q(1-q) \widetilde{L_{q}}+\left(q-\frac{1}{2}\right) \widetilde{E_{q}}$ acts on polynomials of $t$ as multiplication by the factor $\left(t+\frac{d}{2}\right)$. Thus we see that the polynomials $\omega_{k}$ satisfy the recurrence formula:

$$
\omega_{k+1}(t)-q(1-q) k(k+d-1) \omega_{k-1}(t)-[t+(1-q) d-(2 q-1) k] \omega_{k}(t)=0 .
$$

Setting in addition $\omega_{-1}=0$, the formula (4.9) makes sense for all $k \in \mathbb{Z}_{+}(=\{0,1,2, \ldots\})$ and with the initial condition $\omega_{0}=1$ determines the sequence $\omega_{k}$ uniquely. Now we can formulate our first main result.

TheOrem 1. Let ${ }_{2} F_{1}(a, b, c ; x)$ be the Gauss hypergeometric function. The polynomials $\omega_{k}$ defined in Lemma 4 are given by the formula

$$
\omega_{k}(t)=(d)_{k}(1-q)_{2}^{k} F_{1}\left(-t,-k, d ; \frac{1}{1-q}\right),
$$

where $(d)_{k}=d(d+1)(d+2) \ldots(d+k-1)$ denotes the shifted factorial.

Proof. Let us first recall the well-known Gauss recurrence formulae for the hypergeometric function (cf. [1])

$(2 b-c-b x+a x)_{2} F_{1}(a, b, c ; x)+(c-b)_{2} F_{1}(a, b-1, c ; x)+b(x-1)_{2} F_{1}(a, b+1, c ; x)=0$.

After the substitution of parameters

$$
a=-t, \quad b=-k, \quad c=d, \quad x=\frac{1}{1-q},
$$

it coincides with the recurrence equation (4.9). Since by definition $\omega_{0}=1$ and similarly ${ }_{2} F_{1}\left(-t, 0, d ; \frac{1}{1-q}\right)=1$ we obtain the required result.

REMARKs. 1) For $q=1$ the expression for $\omega_{k}$ given in Theorem 1 should be understood as a limit

$$
\omega_{k}(t)=(d)_{k} \lim _{q \mapsto 1^{-}}(1-q)_{2}^{k} F_{1}\left(-t,-k, d ; \frac{1}{1-q}\right) .
$$


2) Other relations for ${ }_{2} F_{1}$ give other formulae fulfilled by $\omega_{k}$. For example from the equation

$(2 a-c-a x+b x)_{2} F_{1}(a, b, c ; x)+(c-a)_{2} F_{1}(a-1, b, c ; x)+a(x-1)_{2} F_{1}(a+1, b, c ; x)=0$ we obtain the difference equation (4.8).

With a suitable change of variable it is possible to obtain an explicit expression for the generating function for this family of polynomials. Recall that given a sequence of polynomials $\left(g_{k}(\lambda)\right)_{k=0}^{\infty}$, the function

$$
G(\lambda, s)=\sum_{k=0}^{\infty} g_{k}(\lambda) s^{k}
$$

is said to be a generating function for $\left(g_{k}(\lambda)\right)_{k=0}^{\infty}$.

Now make the substitution $t+t_{0}=-\frac{i}{\alpha} \lambda$, where $\alpha=(q(1-q))^{-1 / 2}$ and $t_{0}=(1-q) d$ and suitably renormalize the sequence of polynomials by setting

$$
g_{k}(\lambda)=g_{k}(q, d ; \lambda)=\frac{i^{k} \alpha^{k}}{k !} \omega_{k}(t), \quad \text { where } \quad \lambda=i \alpha\left(t+t_{0}\right) .
$$

Setting in addition $s_{0}=i \alpha\left(q-\frac{1}{2}\right)$, we can rewrite the recurrence equation (4.9) in the form

$$
(k+2) g_{k+2}(\lambda)+(k+d) g_{k}(\lambda)+\left[-\lambda+2 s_{0}(k+1)\right] g_{k+1}(\lambda)=0 .
$$

Multiplying both sides of (4.11) by $s^{k+1}$ and taking the sum over all nonnegative $k$, we obtain an equation satisfied by the generating function for $\left(g_{k}(\lambda)\right)_{k=0}^{\infty}$ :

$$
\left(1+2 s_{0} s+s^{2}\right) \frac{\partial G}{\partial s}(\lambda, s)=(-d s+\lambda) G(\lambda, s) .
$$

Performing integration over $s$, we arrive at the following result.

TheOrem 2. Given $q \in[0,1]$ and $d \in \mathbb{N}$ the function

$$
G(q, d ; \lambda, s)=\frac{\exp \left[\frac{2}{\alpha}\left(\lambda+d s_{0}\right) \arctan \left(\frac{2}{\alpha}\left(s+s_{0}\right)\right)\right]}{\left[\left(s+s_{0}\right)^{2}+\frac{\alpha^{2}}{4}\right]^{\frac{d}{2}}}
$$

has the following expansion:

$$
G(q, d ; \lambda, s)=\sum_{k=0}^{\infty} g_{k}(\lambda) s^{k} .
$$

Now we discuss in more detail the classical case $q=\frac{1}{2}$ of the Weyl ordering. By a straightforward inductive argument we obtain

Lemma 7. The polynomials $\omega_{k}\left(\frac{1}{2}, d ; t\right)$ are even for even $k$, and odd for odd $k$.

As a consequence, after the substitution (4.10), the polynomials $g_{k}(\lambda)$ are real valued as functions of $\lambda=i \alpha\left(t+t_{0}\right)$. Moreover, the previous results simplify to the following form.

COROLlaRY 2. The sequence $\left(g_{k}\right)$ satisfies the recurrence equation

$$
(k+2) g_{k+2}(\lambda)=\lambda g_{k+1}(\lambda)-(k+d) g_{k}(\lambda),
$$


and its generating function $G(d ; \lambda, s)=G\left(\frac{1}{2}, d ; \lambda, s\right)$ is given by

$$
G(d ; \lambda, s)=\frac{e^{\lambda \arctan s}}{\left(\sqrt{s^{2}+1}\right)^{d}}, \quad k=0,1, \ldots
$$

As we mentioned before, a special case (with $d=1$ and $q=\frac{1}{2}$ ) of these results was obtained previously by C. M. Bender et al. in [2], and more systematically by T. H. Koornwinder in [10]. In particular the connection of $g_{k}$ with the continuous Hahn polynomials is observed there, and in the former paper also the formula for the generating function identical with the case of $d=1$ of our formula (4.14) was given. However, neither the case of $q=\frac{1}{2}$ and $d>1$ nor other cases of our results were mentioned there.

The next result identifies the polynomials $g_{k}(\lambda)$ with known classes of orthogonal polynomials.

Recall (see [10]) that the continuous Hahn polynomials $p_{k}(x ; a, b, c, d)$ are defined by $p_{k}(x ; a, b, c, d)=i^{k} \frac{(a+c)_{k}(a+d)_{k}}{k !}{ }_{3} F_{2}(-k, k+a+b+c+d-1, a+i x ; a+c, a+d ; 1)$ where ${ }_{3} F_{2}$ is the generalized hypergeometric function (see [1]), while the Meixner-Pollaczek polynomials $P_{n}^{(a)}(x ; \phi)$ are given by

$$
P_{n}^{(a)}(x ; \phi)=e_{2}^{i n \phi} F_{1}\left(-n, a+i x, 2 a ; 1-e^{-2 i \phi}\right) .
$$

The proof of the next result is a matter of standard calculation, when one takes into account the following formula connecting hypergeometric functions, quoted in [10]:

$$
{ }_{2} F_{1}(-n, 2 a+2 i x, 4 a ; 2)={ }_{3} F_{2}\left(-n, n+4 a, a+i x ; 2 a, 2 a+\frac{1}{2} ; 1\right) .
$$

THEOREM 3. For an arbitrary $d \in \mathbb{N}$ and for $q=\frac{1}{2}$ the polynomials $g_{k}(\lambda)=g_{k}\left(\frac{1}{2}, d ; \lambda\right)$ given by (4.10) can be identified as follows:

$$
g_{k}(\lambda)=\frac{(d)_{k}}{\left(\frac{d}{2}\right)_{k}\left(\frac{d}{2}+\frac{1}{2}\right)_{k}} p_{k}\left(\frac{\lambda}{4} ; \frac{d}{4}, \frac{d}{4}+\frac{1}{2}, \frac{d}{4}, \frac{d}{4}+\frac{1}{2}\right)
$$

and also

$$
g_{k}(\lambda)=\frac{(d)_{k}}{k !} P_{k}^{\left(\frac{d}{2}\right)}\left(\frac{\lambda}{2} ; \frac{\pi}{2}\right) .
$$

Moreover, they are orthogonal on $(-\infty, \infty)$ with respect to the measure $\rho(\lambda) d \lambda$, where $\rho(\lambda)=\left|\Gamma\left(\frac{d}{2}+i \frac{\lambda}{2}\right)\right|^{2}$.

Let us point out that the polynomials $\omega_{k}(t)$ do not share certain properties of $g_{k}(\lambda)$. In particular by rewriting the recurrence equation (4.9) in the form

$$
\omega_{k+1}(t)=\left(A_{k} t+B_{k}\right) \omega_{k}(t)-C_{k} \omega_{k-1}(t)
$$

we see that

$$
A_{k-1} A_{k} C_{k}=-q(1-q) k(k+d-1) \leq 0 .
$$

Thus the necessary condition for the existence of a positive measure, with respect to which $\omega_{k}$ would be orthogonal, is violated.

Also in the general case of nonsymmetric ordering corresponding to $q \neq \frac{1}{2}$ the general picture is not so clear and satisfactory as in the symmetric case. From (4.8) one could 
expect that the set $\left\{\omega_{k}\right\}$ can be described as a subset of two known families of polynomials - Krawtchouk $\left(K_{n}\right)$ and Meixner $\left(M_{n}\right)$. In fact by comparing parameters we see that

$$
{ }_{2} F_{1}\left(-t,-k, d ; \frac{1}{1-q}\right)=K_{k}(t, 1-q,-d)=M_{k}\left(t, d,-\frac{1-q}{q}\right) .
$$

However, there is again a problem with orthogonality, since the parameters occurring in these formulae fall outside the range required by the orthogonality condition (see [1] and $[12])$.

Acknowledgements. The ideas of this paper arose during the doctoral studies of the first named author at the Department of Mathematical Methods of Physics, Faculty of Physics, University of Warsaw under the supervision of the second named author. Various parts of the results contained in this paper were presented at the Banach Center "Workshop on Lie groups and Lie algebras" in Bdlewo, September 2000 and Biaowieża Workshop on Geometric Methods in Physics, July 2001. A fuller presentation of the topic, discussing connections to other classes of polynomials is contained in the doctoral dissertation of the first named author.

Part of the work on this paper was done while the second named author enjoyed the stay at the Humboldt University in Berlin thanks to the support of SFB 288 Differential geometry and quantum physics. This support and the hospitality of Professor Dr. Thomas Friedrich are gratefully acknowledged.

After this work has been finished, Professor M. Engliš has pointed out to us the connection of our construction of the $q$-ordering map $\mathcal{O}_{q}$ with the extended symbol calculus discussed by A. Unterberger in Encore des classes de symboles, Séminaire GoulaouicSchwartz 1977/1978, Exposé No. 6, École Polytech., Palaiseau 1978. We intend to investigate this relation in more detail in a later work and we thank him for this indication.

Last but not least, we are grateful to the referee for his very careful reading of the manuscript and many remarks which led to an improvement of the paper.

Note added in proof: After the completion of this paper A. Verçin has kindly pointed out to us his papers Ordered products, $W_{\infty}$-algebra, and two-variable, definiteparity, orthogonal polynomials, J. Math. Phys. 39 (1998), 2418-2427, and Metaplectic covariance of the Weyl-Wigner-Groenewold-Moyal quantization and beyond, Ann. Phys. 266 (1998), 503-523, where some of the above results on the connection of the Hahn polynomials with the ordering of operators in the Weyl algebra are also obtained from different premises.

\section{References}

[1] G. E. Andrews, R. Askey and R. Roy, Special Functions, Cambridge University Press, Cambridge, 1999.

[2] C. M. Bender, L. R. Mead and S. Pinsky, Continous Hahn polynomials and the Heisenberg algebra, J. Math. Phys. 28 (1987), 509-513.

[3] C. M. Bender and G. V. Dunne, Polynomials and operator orderings, J. Math. Phys. 29 (1988), 1727-1731. 
[4] L. C. Biedenharn and J. D. Louck, An intrinsically self-conjugate boson structure: the symplecton, Ann. Phys. (N.Y.) 63 (1971), 459-475.

[5] J. Faraut, Analyse harmonique et fonctions speciales, in: J. Faraut and K. Harzallah, "Deux Cours d'Analyse Harmonique", École d'Été d'Analyse Harmonique de Tunis 1984, Birkhäuser Verlag, Basel, 1987.

[6] G. B. Folland, Harmonic Analysis in the Phase Space, Princeton University Press, Princeton, 1989.

[7] D. Geller, Spherical harmonics, the Weyl transform and the Fourier transform on the Heisenberg group, Can. J. Math. 36 (1984), 615-684.

[8] R. Howe and E. C. Tan, Non-abelian Harmonic Analysis: Applications of $S L(2, \mathbb{R})$, Springer-Verlag, New York, 1992.

[9] M. Ikeda and N. Seto, On expansion theorems in terms of spherical functions for unitary group, I, Math. Jap. 13 (1968), 149-157.

[10] T. H. Koornwinder, Meixner-Pollaczek polynomials and the Heisenberg algebra, J. Math. Phys. 30 (1989), 767-769.

[11] M. A. Lohe, L. C. Biedenharn and J. D. Louck, Tensor operator formulation of Weyl ordered polynomials, Phys. Rev. D 43 (1991), 617-619.

[12] A. F. Nikiforov, S. K. Suslov and V. B. Uvarov, Classical Orthogonal Polynomials of a Discrete Variable on Nonuniform Lattices, Springer Verlag, New York, 1991.

[13] A. Strasburger, On the decomposition of the oscillator representation, in: Quantization and coherent states methods, in: Proc. of the XI Workshop on Geometric Methods in Physics, S. T. Ali et al. (eds.), World Scientific, Singapore, 1993, 127-133.

[14] A. Strasburger, Weyl algebra and a realization of the unitary symmetry, Rend. Circ. Mat. Palermo Ser. II, Suppl. 54 (1998), 101-111.

[15] A. Strasburger, On algebras of creation and annihilation operators, in: CRM Proc. Lecture Notes 22, Algebraic Methods and $q$-Special Functions, L. Vinet and J. F. van Diejen (eds.), Amer. Math. Soc., Providence, Rhode Island, 1999, 267-276.

[16] N. Ja. Vilenkin and A. U. Klimyk, Representations of Lie Groups and Special Functions, Kluwer Academic Publishers, Dordrecht, 1992.

[17] K. B. Wolf, The Heisenberg-Weyl ring in quantum mechanics, in: Group Theory and its Applications, Vol. III, E. M. Loebl (ed.), Academic Press, New York, 1975, 189-247. 